\title{
Kinetic modeling of relaxation phenomena after photodetachment in a rf electronegative $\mathrm{SiH}_{4}$ discharge
}

\author{
M. Yan, * A. Bogaerts, and R. Gijbels \\ Department of Chemistry, University of Antwerp (UIA), Universiteitsplein 1, B-2610 Wilrijk-Antwerp, Belgium \\ W. J. Goedheer \\ FOM-Institute for Plasma Physics “Rijnhuizen,'” P.O. Box 1207, 3430 BE Nieuwegein, The Netherlands \\ (Received 4 August 2000; published 22 January 2001)
}

\begin{abstract}
The global relaxation process after pulsed laser induced photodetachment in a rf electronegative $\mathrm{SiH}_{4}$ discharge is studied by a self-consistent kinetic one-dimensional particle-in-cell-Monte Carlo model. Our results reveal a comprehensive physical picture of the relaxation process, including the main plasma variables, after a perturbation up to the full recovery of the steady state. A strong influence of the photodetachment on the discharge is found, which results from an increase of the electron density, leading to a weaker bulk field, and hence to a drop in the high energy tail of the electron energy distribution function (EEDF), a reduction of the reaction rates of electron impact attachment and ionization, and a subsequent decrease of the positive and negative ion densities. All the plasma quantities related to electrons recover synchronously. The recovery time of the ion densities is about 1-2 orders of magnitude longer than that of the electrons due to different recovery mechanisms. The modeled behavior of all the charged particles agrees very well with experimental results from the literature. In addition, our work clarifies some unclear processes assumed in the literature, such as the relaxation of the EEDF, the evolution of the electric field, and the recovery of negative ions.
\end{abstract}

DOI: $10.1103 /$ PhysRevE.63.026405

PACS number(s): 52.25.Dg, 52.80.-s

\section{INTRODUCTION}

Laser induced photodetachment is a widely used technique to measure the density, thermal energy and velocity of negative ions in electronegative rf discharges (e.g., Refs. [1-8] and references therein). This method applies photons to transform negative ions by photodetachment into electrons and neutrals, and subsequently an increase of the electron density is detected by microwave cavity resonance spectroscopy $[1,2]$ or by the optogalvanic method [3] or by using a probe $[4-6,8]$. From the measured electron density, the negative ion density can be deduced, since the loss of negative ions is equal to the production of the electrons. From the investigation of the negative ion recovery, combined with a ballistic approximation equation, the thermal energy of the negative ions is obtained [5]. In addition, from the duration of the plateau in the probe signal, an estimate of the negative ion velocity can be made [6].

Since the photodetachment method relies on the detection of the electron density, it is very important to understand the relaxation mechanism of the extra electron density and energy. Since electron relaxation will influence the ionization and attachment rates and the production of positive and negative ions, and hence the whole discharge, it is also important to understand the global relaxation characteristics. Many experimental studies have been performed to investigate relaxation phenomena, such as the decay of the extra electron density (e.g., Refs. $[1,4,5]$ ) and the recovery of the ion densities (e.g., Refs. [5,9]), etc. However, unlike experi-

\footnotetext{
* On leave from Dept. of Engineering Physics, Tsinghua University, China. Email address: yan@uia.ua.ac.be
}

ments, only a few theoretical studies were made [10-14], which analyzed only particular aspects of the relaxation. No complete physical picture of the relaxation process was given in previous theoretical studies due to the lack of effective kinetic methods to describe the whole relaxation process in electronegative discharges.

Passchier and Goedheer [10] used a fluid model to investigate the relaxation phenomena of extra electrons after a laser induced photodetachment pulse in $\mathrm{CF}_{4} \mathrm{rf}$ discharges. The relaxation of the electron density, the reaction rates of electron impact collisions such as ionization and attachment, and the electron flux to the wall were studied. However, a fluid model cannot describe the electron behavior accurately and kinetically, and some simplifying assumptions regarding the fast relaxation of the electron energy, and hence a quick recovery of the reaction rates (only several rf cycles at a few hundred mTorr), were used in their model. As a result, they found that the relaxation time of the extra electrons is several times longer than the experimental value. The authors of Ref. [11] investigated the effect of laser induced photodetachment in an oxygen rf discharge by using a relaxation continuum model, which is also based on the fluid approach [15]. They considered the effect of continuous laser irradiation or of a long laser pulse on the discharge. They also assumed that the electron energy distribution function (EEDF) relaxation is very short (just one rf cycle), and ignored the influence of the photodetached electron energy on the EEDF. They only took into account the influence of the change of the electron number density on the electric field. Since the electron density and the EEDF are the key parameters determining the relaxation of the whole rf discharge, and since the relaxation process is accompanied by a strong kinetic variation, the assumption concerning the fast recovery of the EEDF in the above described fluid models is not justified. 
The authors of Ref. [12] used a hybrid fluid-kinetic approach to study the overshoot in the time dependence of the electron density perturbation in low pressure hydrogen plasma. In this model, electrons and positive ions are described by the fluid theory, while negative ions are treated within the ballistic kinetic theory without considering the effects of the electric field. The authors of Ref. [13] considered the effects of a self-consistent electric field on negative ions, and used a self-similar method (well known in hydrodynamics) to examine the counterflow of the negative plasma species. Both studies assumed that electrons inside and outside the laser impact region mix to an equilibrium state only with a density inhomogeneity, i.e., the electrons are ruled by the Boltzmann law. Recently Ivanov et al. [14] studied the initial perturbation in a plasma with negative ions. They used an analytical method, namely, a kinetic approach based on the Fourier-Laplace transform and on the linearized kinetic equation for electrons and positive and negative ions. They derived expressions for the electron density as a function of time and coordinates, and explained the appearance and disappearance of a dip in the electron density profile. Since the authors of Refs. [12-14] mainly used analytical methods to describe the relaxation process, many assumptions had to be made, and only limited situations and separate phenomena were considered. For example, elastic and inelastic collisions were neglected, and they assumed that the initial negative ion density is much less than the electron density, and that electrons mix to an equilibrium state. Consequently, Refs. [12-14] were limited to weak electronegative discharges, to a non-full recovery process (collisions are very important for the full recovery of ions; see below), and to local phenomena.

In the present paper we investigate the complex relaxation mechanism after instantaneous laser induced photodetachment in electronegative rf discharges by a completely selfconsistent kinetic one-dimensional particle in-cell-Monte Carlo (PIC-MC) model developed specifically for discharges in $\mathrm{SiH}_{4}$ [16]. The $\mathrm{SiH}_{4} \mathrm{rf}$ discharge serves as an example to qualitatively demonstrate the general characteristics of the relaxation process after photodetachment in other electronegative discharges. The ratio of the electron density and the negative ion density in $\mathrm{SiH}_{4}$ discharges is in the same range as in $\mathrm{CF}_{4}$ and $\mathrm{Cl}_{2}$ discharges for similar discharge conditions. There are some experiments $[1,3]$ which concerned the study of photodetachment in $\mathrm{CF}_{4}$ and $\mathrm{Cl}_{2}$ discharges. These discharges have physical characteristics similar to those of $\mathrm{SiH}_{4}$ discharges. The present work is a representative study of the main relaxation mechanism after photodetachment, using a well developed code.

We study relaxation process after a perturbation (photodetachment) up to full recovery of the steady state. We focus on the relaxation mechanism, including the evolution of the charged particle densities, the electric field, the EEDF, the average electron energy, and the reaction rates of the electron impact collisions as well as the power dissipation, after the photodetachment. The influence of the pressure and the efficiency of the photodetachment on the relaxation process is discussed. Since experimental data are often the result of a combination of many different effects in a complex system, such as a rf discharge with the interaction between the electric field and the charged particles, theoretical studies can assist in understanding the physical mechanism and clarify the observations in the experiments. A comparison between our simulation results and experimental results from the literature is used to validate our model and to assist in the interpretation of the experimental observations.

\section{MODEL DESCRIPTION}

The PIC-MC method is based on a kinetic description of particle motion in phase space. Charged "superparticles" move in the self-consistent electric field which they generate. A Monte Carlo formalism is used to describe collisions. This method simulates the behavior of the particles on the lowest microscopic level, and it does not make use of many assumptions. Detailed descriptions of the PIC-MC method can be found, e.g., in Refs. [17], [18]. Many speed-up methods $[16,19]$ for obtaining the periodic converged results have been used in the present simulation.

The discharge gas is assumed to be pure silane. For simplification, only three types of charged species, [i.e., electrons $\left(e^{-}\right)$, positive ions $\left(\mathrm{SiH}_{3}^{+}\right)$, and negative ions $\left(\mathrm{SiH}_{3}{ }^{-}\right)$], are taken into account, which is justified by Hamers' measurements [20] and results from fluid modeling [21]. The electron impact collisions included in the model are elastic collisions, vibrational excitation, attachment, dissociation, and ionization [16]. The ion impact collisions are elastic collisions, charge transfer and positive ion-negative ion recombination [16]. The initial conditions and assumptions for the simulations are the following.

(1) A laser pulse is fired after the discharge has reached a periodic steady state, and the pulse duration is neglected.

(2) The negative ion density $\left(\mathrm{SiH}_{3}{ }^{-}\right)$in the laser impact region drops due to the photodetachment, and the same amount of electrons is released by the photodetachment at exactly the same position. The positive ion $\left(\mathrm{SiH}_{3}{ }^{+}\right)$density does not change during the pulse.

(3) The only laser induced reaction is considered to be the photodetachment $\mathrm{SiH}_{3}{ }^{-}+h \nu \rightarrow \mathrm{SiH}_{3}+e^{-}$. The electron affinity of $\mathrm{SiH}_{3}$ is $1.4 \mathrm{eV}$ [22].

(4) All the detached electrons have an energy of $1 \mathrm{eV}$, which is the difference between the photon energy and the electron affinity.

(5) The laser is fired at the beginning of a rf cycle.

(6) The process is considered to be one dimensional. The laser beam is assumed to be infinite in directions parallel to the electrodes.

(7) The background gas density (feed gas) is regarded as uniform and the gas pressure is fixed during the simulation.

In order to compare the influence of both the plasma bulk field and the sheath field on the relaxation phenomena, we choose the laser injection point not at the discharge center but at a position $1 \mathrm{~cm}$ below the grounded electrode (the distance between the two electrodes is $3 \mathrm{~cm}$ ). The laser beam diameter is $2 \mathrm{~mm}$. A sketch of the photodetachment event in the reactor is shown in Fig. 1.

If not specified otherwise, all negative ions in the laser beam are assumed to be photodetached. The applied rf fre- 


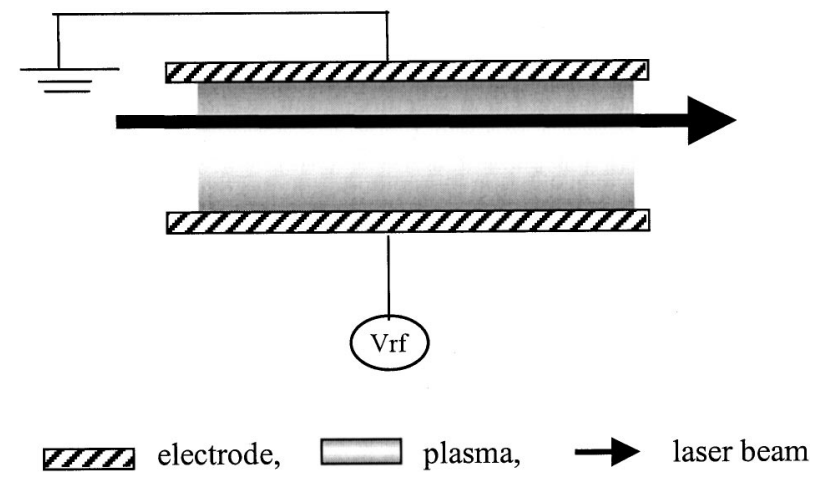

FIG. 1. Sketch of laser photodetachment in the rf discharge.

quency is fixed at $13.56 \mathrm{MHz}$. The power is $7.5 \mathrm{~W}$ which implies an applied voltage of around $300 \mathrm{~V}$. The pressures are 400, 100, and 30 mTorr, respectively, which is in the normal pressure range for thin film deposition in $\mathrm{SiH}_{4}$ discharges and which is also in the same range as in Refs. [1], [10], [11]. We want to study the main relaxation mechanism after photodetachment, which is easier at a higher pressure, where the densities of the charged species are higher, and the phenomena can be studied even at a relatively low input power. Based on the tendency from 400 to 30 mTorr in Table II, the situation of a few mTorr could also be deduced (see below). The secondary electron emission coefficient and the reflection coefficient from the electrodes are assumed to be zero. In the simulations we use 64 spatial grid points and 1000 time steps in one rf cycle. For each type of charged particle (i.e., electrons and $\mathrm{SiH}_{3}{ }^{+}$, and $\mathrm{SiH}_{3}{ }^{-}$) 3000-7000 "superparticles" are followed.

\section{RESULTS AND DISCUSSION}

\section{A. Relaxation of the electron density and energy}

\section{Relaxation of the electron density}

The electron density profiles at zero, ten, 100, and $500 \mathrm{rf}$ cycles after the photodetachment are shown in Fig. 2. "0 $\mu \mathrm{s}$ " means that the sample is taken immediately after the

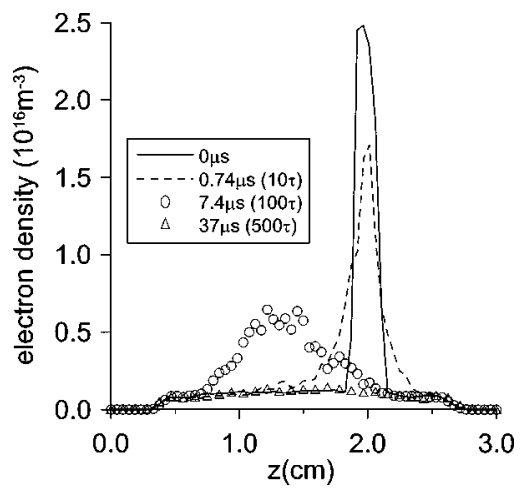

FIG. 2. The calculated electron density profile at zero, ten, 100, and $500 \mathrm{rf}$ cycles after photodetachment, at $400 \mathrm{mTorr}, 13.56 \mathrm{MHz}$, and $7.5 \mathrm{~W}$. The laser impact point is at $1.0 \mathrm{~cm}$ below the grounded electrode (cf. Fig. 1). $\tau$ is the time period of one rf cycle.

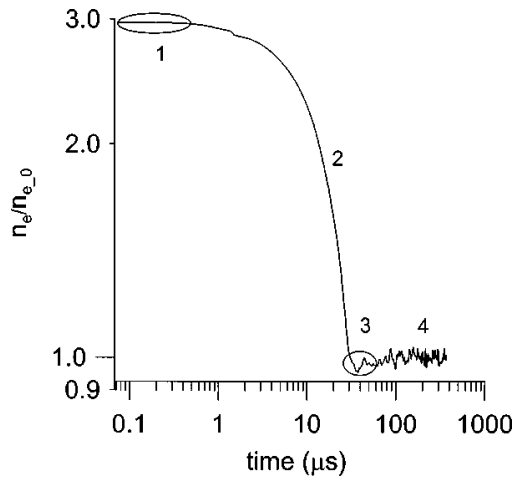

FIG. 3. The calculated evolution of the ratio (on a logarithmic scale) of the integrated electron density and its original value. The conditions are the same as in Fig. 2. The ellipse in part 1 marks the plateau at the beginning. The ellipse in part 3 marks the overshoot.

photodetachment. It appears that the relaxation process of the extra electrons proceeds as follows.

(1) The extra electrons diffuse very rapidly due to the strong electron density gradient in the laser impact region. Since we assume the laser impact point close to the grounded electrode, the diffusion is stronger towards the weak field side, i.e., the plasma center.

(2) The peak of the extra electron density decreases with time; the profile becomes broader than the width of the laser beam, and the peak shifts to the plasma center (see the curve at the 100th rf cycle). When the peak decreases at the laser impact point, it can split into two peaks after a certain relaxation time; this effect is more pronounced at low pressure, but is not shown here. This dip in the density profile was discussed in Ref. [14].

(3) After $500 \mathrm{rf}$ cycles, the electron density profile has recovered almost completely. It is clear from Fig. 2 that the relaxation of the local effect of photodetachment is not only a local behavior (i.e., at the laser impact point) but also a global process.

The relaxation behavior of the electron density was measured in experiments [4,5], where the photodetached electron current was collected by probes (see Fig. 2 in Ref. [4] and Fig. 3 in Ref. [5]). The typical electron current signal vs time reflects the relaxation process of electrons. The extra electron current in Refs. [4,5] shows a more or less steady state profile at the beginning, then it drops quickly and after an overshoot back to the original value. This relaxation behavior is quite similar to the picture obtained from our simulation. Figure 3 shows the global evolution of the ratio of $n_{e} / n_{e_{-} 0}$. $n_{e}$ is the integral of electron density over the discharge after the photodetachment, and $n_{e_{-} 0}$ is its original value. It can be seen that there are four steps for the electron density relaxation. During the first several rf cycles the value of $n_{e} / n_{e_{-} 0}$ is rather constant. Then, in the second stage, it drops with a more or less constant speed. The third step shows an overshoot, after which the electron density stops to drop; finally, the steady state value is recovered. The recovery time of the electron density $\left(\tau_{e}\right)$ is about $37 \mu$ s for the 400-mTorr case. These relaxation phenomena can be understood from the ob- 
servation that at the very beginning the extra electrons need time to diffuse away from the laser impact point, and the later fast drop occurs when the electrons start to leave the discharge. The overshoot is related to a loss of positive ions accompanying the electrons and to less production, causing the total electron density to decrease below its initial periodic steady state value in order to maintain charge neutrality, before returning to the original value $[6,12,23]$. The overshoot in the electron density in the probe experiments mentioned above is much stronger than that obtained in our simulation. Further, in the experiment with microwave cavity resonance spectroscopy [1], this overshoot cannot be seen. The strong overshoot in the experiments could result from the influence of the biased probe which can disturb the plasma due to its applied voltage.

Note that part 2 in Fig. 3 has less fluctuation than the steady state part (marked by 4 in Fig. 3). This is because in part 2 the relaxation of the electrons is dominated by the strong electron diffusion. Consequently, the fluctuation of the electron impact collisions in part 2 is not as apparent as in part 4.

Note that $\tau_{e}$ of $37 \mu \mathrm{s}$ in our situation is quite different from the experimental value [4,5], which is less than $1 \mu \mathrm{s}$. However, the recovery of electrons after photodetachment depends on the production by ionization, the loss by attachment, and the diffusion to the wall. After the photodetachment has taken place, the effect of the ionization and attachment on the electron relaxation can be neglected due to the reduced ionization rate $\left(R_{\text {ion }}\right)$ and attachment rate $\left(R_{\text {att }}\right)$ (see Fig. 7 below) and due to the strong electron density gradient. Therefore, the diffusion process dominates the relaxation of the electron density, and $\tau_{e}$ depends mainly on the diffusion, and hence on the background gas pressure. In the experiments $[4,5]$ the pressure is several mTorr, while our simulation results are obtained at 400 mTorr. Moreover, when the pressure increases from 3 to $7 \mathrm{mTorr}$ in the experiments, $\tau_{e}$ increases from $600 \mathrm{~ns}$ to $1.1 \mu \mathrm{s}$ [5]. A similar strong relationship between $\tau_{e}$ and the pressure is found in our simulation (see Table II below). When the pressure decreases from 400 to $30 \mathrm{mTorr}, \tau_{e}$ reduces from 37 to $5 \mu \mathrm{s}$. Some other factors can also cause the difference in $\tau_{e}$. First, the signal collected by the probe will not reflect the total extra electron density because it has been pointed out that the probe is only sensitive to density fluctuations up to $1 \mathrm{~mm}$ from its axis, and will not measure the density fluctuations that occur at larger distances [6]. However, as shown in Fig. 2, the extra electrons can diffuse far from the laser impact region. Second, we only consider the 1D case; relaxation in the radial direction can also reduce $\tau_{e}$. Third, different gases are considered, in the simulations $\mathrm{SiH}_{4}$ and in the experiments $\mathrm{H}_{2}$.

The global decay behavior of the extra electrons was also compared with another experimental result [1], where the extra electrons were measured by the microwave cavity resonance spectroscopy method. A frequency shift due to the extra free electrons created by the photodetachment was measured, and consequently the extra electron density $\Delta n_{e}$ was deduced according to the formula [1]

$$
\frac{\Delta f^{\prime}}{f_{0}}=\frac{1}{2} \frac{\left(\Delta n_{e} e^{2} / m_{e} \varepsilon_{0} \omega^{2}\right)\left\{O /\left[1+\left(\nu_{m}^{2} / \omega^{2}\right)\right]\right\} \int_{-R}^{R} E^{2} d r}{\iiint_{\text {cavity }} E^{2} d^{3} r},
$$

where $\Delta f^{\prime}$ is the frequency shift caused by the extra detached electrons, $f_{0}$ is the resonance frequency of the empty cavity, $m_{e}$ is the mass of an electron, $e$ is the elementary charge, $\omega$ is the angular frequency of the microwaves, $\nu_{m}$ is the electron collision frequency, $E$ is the microwave electric field distribution in the cavity, $O$ is the area of the laser beam, $\Delta n_{e}$ is the extra electron density, and $R$ is the inner radius of the cavity. The authors found that the extra electron density decays with two clearly different time scales (see the 100-mTorr $\mathrm{CF}_{4}$ discharge in Fig. 3 of Ref. [1]): (i) a fast decay in several $\mu$ s and (ii) a slow decay in several tens of $\mu \mathrm{s}$ at 100 mTorr. The authors explained this behavior by the fact that the first fast decay period is related to a lower power input caused by the photodetachment, which leads to a favoring of attachment over ionization in the discharge, resulting in a decrease of the net generation rate of the electrons. For the second period, the authors assumed that the extra electrons have relaxed to have the same temperature and spatial distribution as the rest of the electrons. The total number of electrons is then still higher than before the laser pulse, and will tend to return to the original value on a diffusion timescale.

The total electron recovery time shown in Ref. [1] is of the same order of magnitude as our simulation, but we did not observe the two decay speeds. In our simulations, we did not find that the attachment events are much favored with respect to the ionization (see Fig. 7 below) in the first period of the experiment mentioned above after the photodetachment. From Fig. 2, one can see that the assumption of decay for the second period in Ref. [1] is not valid, i.e., the profile of the extra density cannot be considered to be as homogeneous as the original electron density. The homogeneouslike profile of the electron density occurs only when the profile has recovered almost completely. We think that the different decay behaviors between the simulation and the experiment could be a result of simplifying formula (1) in Ref. [1], from a form with an integration over the whole cavity to the present one. It is also possible that another relaxation mechanism plays a role, like the $e-e$ collisions that are not included in our model, and that at the beginning the electron density in the laser impact zone is very high. Moreover, the function of the attachment cross section vs energy for the $\mathrm{SiH}_{4}$ gas has a peaked shape [22], which is quite different from the $\mathrm{CF}_{4}$ gas investigated in Refs. [1,10].

\section{Relaxation of the electron energy}

Since the electrons obtain energy from the electric field, it is interesting to check first the evolution of the electric field and potential after the photodetachment. Figure 4 shows the electric potentials at zero, ten, 100, and $500 \mathrm{rf}$ cycles after the photodetachment. At the beginning the potential tends to 


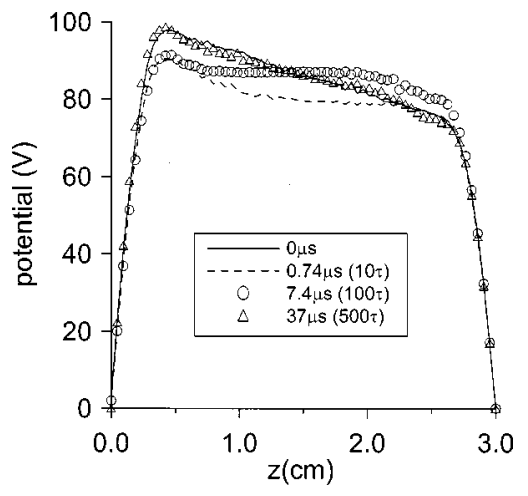

FIG. 4. The calculated evolution of the electric potential distribution in the discharge. The conditions are the same as in Fig. 2.

be flat (from zero to ten rf cycles), i.e., a weak electric field, and after a long time (37 $\mu$ s, i.e., 500 rf cycles), the potential returns to its original shape. The reason for the decrease of the field is the increase of the electron density, which increases the conductivity. The continuity of the current requires a small potential difference in order to compensate for the increase of the conductivity. In Ref. [6] the authors analyzed the electric field after the laser shot. They assumed a change from a field-free situation to a nonzero electric field, which reduced the diffusion flux of electrons and accelerated the filling flux of negative ions. Our result clarifies the behavior of the field, that is, the evolution from a relatively strong field to a weak one. This tendency toward a weaker electric field corresponds to a change of the electric field at the transition from an electronegative discharge to an electropositive discharge. Indeed, after photodetachment, the negative ion density drops while the electron density increases; hence the discharge resembles an electropositive one. This decrease of electric field also agrees with a statement from Ref. [3]

The evolution of the electric field results in a special behavior of the total power dissipation in the discharge. Figure 5 shows the total power dissipation vs time. The power evolves in a three-period process. In the first several rf cycles after the photodetachment, the electric field does not change very much, and the power dissipation remains the same as the original value, which corresponds to a peak at the begin-

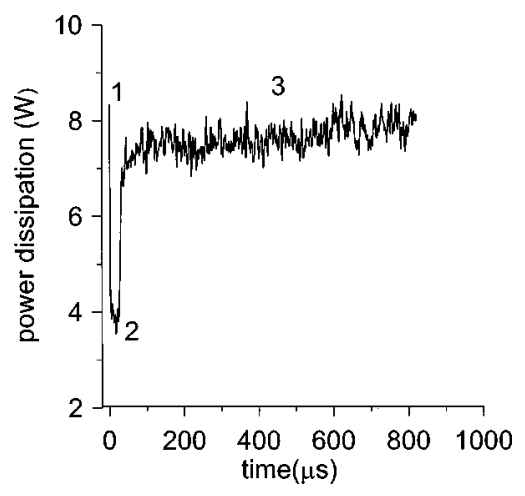

FIG. 5. The calculated evolution of the power dissipation in the discharge after photodetachment. The conditions are the same as in Fig. 2. The sampled time step is ten rf cycles.

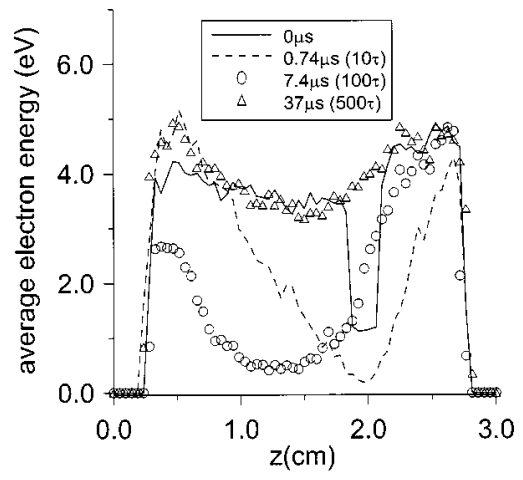

FIG. 6. The calculated evolution of the average electron energy profile after the photodetachment. The conditions are the same as in Fig. 2.

ning around $t=0$ (see the part marked 1 in Fig. 5). Note that the sampled time step for this curve is ten rf cycles (740 ns). With the electric field weakening, the power dissipation drops (almost a factor of 2; see the part marked 2 in Fig. 5) and after about $500 \mathrm{rf}$ cycles it recovers following the evolution of the electric field (see the part marked 3 in Fig. 5).

Figure 6 shows the averaged electron energy profile at one, ten, 100 and 500 rf cycles after the photodetachment. It reflects the characteristics of the relaxation of the electron energy.

(1) In the first several tens of rf cycles after the photodetachment, the average electron energy in the laser impact region does not increase immediately but decreases further. The reason for this is that the fast electrons lose energy due to all kinds of collisions, and a large amount of $1-\mathrm{eV}$ electrons will also lose energy due to vibrational excitation collisions (the threshold energies are at 0.11 and $0.27 \mathrm{eV}$, respectively). In addition, the slow electrons cannot be heated immediately because of the weakened electric field after the photodetachment (see Fig. 4).

(2) Following the diffusion of the slow electrons, the averaged electron energy decreases in the whole discharge, and the minimum point of the energy coincides in time and position with the movement of the maximum in the electron density (see Fig. 2).

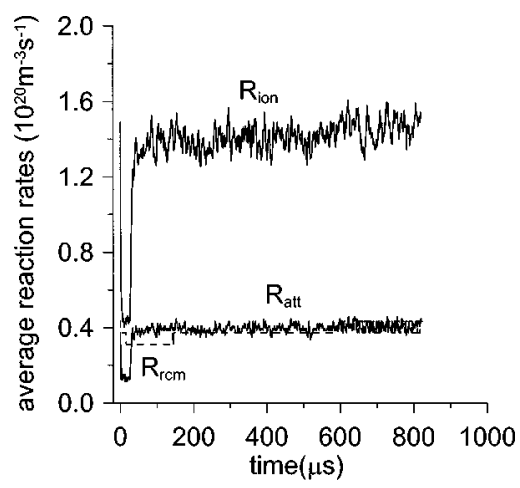

FIG. 7. The calculated evolution of the ionization rate $\left(R_{\text {ion }}\right)$, the attachment rate $\left(R_{\text {att }}\right)$, and the ion-ion recombination rate $\left(R_{\mathrm{rcm}}\right)$ after the photodetachment. The conditions are the same as in Fig. 2. The sampled time step is ten rf cycles. 
(3) The average electron energy recovers with the same characteristic time as the electron density.

From the evolution of the average electron energy, one can deduce that the reaction rates of attachment and ionization should have a similar behavior. In Fig. 7, we present the evolution of the space and rf period averaged $R_{\text {ion }}$ and $R_{\text {att }}$. It can be seen that they show similar behaviors. This behavior of the evolution of $R_{\text {ion }}$ and $R_{\text {att }}$ coincides with the power dissipation (see above). The evolution of the rates can be divided into three periods. In the first period, only several $\mathrm{rf}$ cycles, the reaction rates have not changed because at that moment the fast electrons have not been consumed completely and the electric field has not changed too much. In the second period, the fast electrons are consumed in collisions and loss to the walls, and the electric field becomes weaker, so that the electron energy drops, and hence so do $R_{\text {ion }}$ and $R_{\text {att }}$. In the third period (after 500 rf cycles), with the electron density and the electric field recovering to the original values (see Figs. 2 and 4), the electrons are heated up and the reaction rates also return to their original values.

We do not observe that $R_{\text {att }}$ increases over $R_{\text {ion }}$; this would lead to a long time scale for the first decay period (see Figs. 4 and 5 in Ref. [10]); this was also used as an assumption to explain the electron density decay in Ref. [1]. We think that the decrease of the electron energy is due to the generation of cold electrons, which have an energy below the threshold of both the attachment and the ionization. Therefore, the tail of the EEDF decreases as the inelastic processes go on for a while, and, with this, the rates of both attachment and ionization decrease with more or less the same factor. Furthermore, we think that the same qualitative influence of the electric field on $R_{\text {att }}$ and $R_{\text {ion }}$ should also lead to a similar evolution behavior of the rates, although the behavior of the attachment cross section vs the energy can be different for different gases. The different behavior of the attachment and ionization rate in Ref. [10] (which shows that the total attachment does not change, while the ionization at the beginning decreases a great deal), is actually due to the use of the average electron energy instead of the full EEDF to describe these rates in the fluid model.

As mentioned above, in previous simulations of laser induced photodetachment $[10,11]$, the authors assumed that the EEDF would return to its original shape in a few rf periods. Consequently, the direct influence of the photodetachment on the EEDF was ignored in these works. However, from Fig. 6, it becomes clear that at $400 \mathrm{mTorr}$ it takes several hundred of rf cycles for the average electron energy profile to go back to its original value due to the weakened electric field.

Figure 8 shows the EEDF of the total number of electrons in the discharge at zero, ten, 100, and $500 \mathrm{rf}$ cycles after the photodetachment. The EEDF (arb. units) has not been normalized because we want to see the variation of the electron density in energy space at different moments. One can see that there is a jump at $1 \mathrm{eV}$, which reflects the extra electrons appearing due to the photodetachment. Then this peak moves to very low energies. The Inset in Fig. 8 shows the evolution of the peak during the first rf cycle after the photodetachment. In this first rf cycle the peak becomes broader with

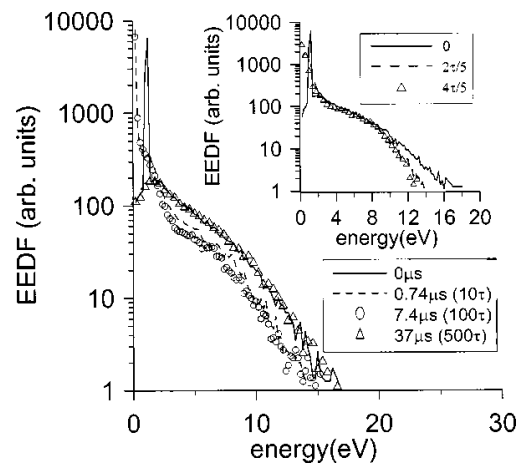

FIG. 8. The calculated evolution of the EEDF in the discharge after photodetachment. The small inserted picture shows the EEDF in the first rf cycle after photodetachment. The conditions are the same as in Fig. 2.

time. The vibrational excitation collisions broaden the peak toward low energies, and the electrical heating broadens it toward higher energies. With the decrease of the heating due to the weakened electric field, the peak gradually moves toward very low energies. In addition, the weak electric field also leads to a significant decay of the high energy tail. From Fig. 8, it can be concluded that the EEDF has been changed strongly. The recovery of the EEDF cannot be finished in one rf cycle, as assumed in previous work $[10,11]$, and in fact it recovers synchronously with the electron density profile. Our result shows that it takes several hundred rf cycles for the EEDF to recover at a pressure of several hundred mTorr (In Refs. $[10,11]$ the gas pressure varies from several hundred mTorr to 1 Torr.)

\section{B. Relaxation of negative ions $\left(\mathrm{SiH}_{3}{ }^{-}\right)$}

Figure 9 shows the negative ion density profile $\left(\mathrm{SiH}_{3}{ }^{-}\right)$at zero, ten, 100, and 500 rf cycles after the photodetachment. The dip in the negative ion density profile in the laser impact region becomes shallower in time due to the inward diffusion from the surrounding zones, while the density in the other part of the profile, outside the dip, decreases gradually (see the curve at the tenth rf cycle). Before the local negative ion density recovers completely, it goes down again with the global density (compare the local density value at the 100th and 500th rf cycles in Fig. 9). These phenomena show that at

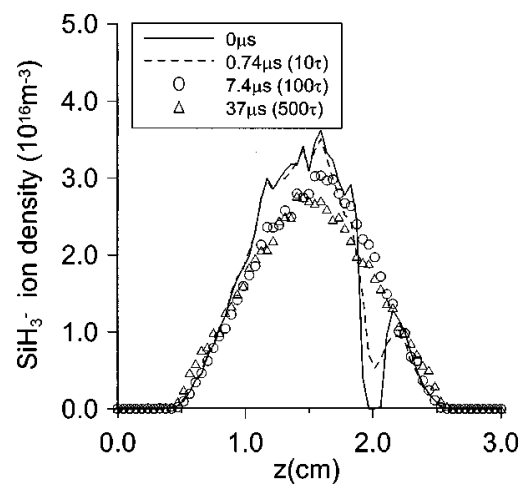

FIG. 9. The calculated evolution of the $\mathrm{SiH}_{3}{ }^{-}$ion density profile after photodetachment. The conditions are the same as in Fig. 2. 


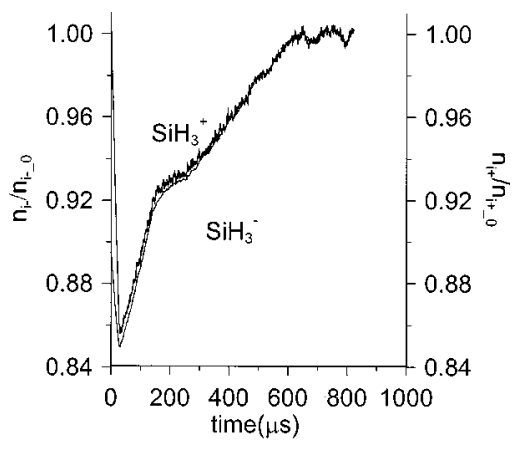

FIG. 10. The calculated evolutions of the ratio of the integrated density of $\mathrm{SiH}_{3}{ }^{-}$and $\mathrm{SiH}_{3}{ }^{+}$, and their original values. The conditions are the same as in Fig. 2. The sampled time step is ten rf cycles.

the beginning the diffusion filling the dip lowers the global density profile, and in the meanwhile the production of negative ions by the electron attachment also decreases strongly; hence there is no compensation for this "photodetachment loss" of negative ions. Only after $500 \mathrm{rf}$ cycles is the attachment rate recovered (see Fig. 7), and the total negative ion density begins to recover as well.

The full recovery process of the space averaged negative ion density is shown in Fig. 10 as the ratio of the integrated $\mathrm{SiH}_{3}{ }^{-}$ion density $\left(n_{i-}\right)$ and its integrated initial value $\left(n_{i-}{ }_{0}\right)$. It can be seen that the $\mathrm{SiH}_{3}{ }^{-}$ion density first decreases with the electron relaxation (see Fig. 3), and starts to recover when the electron density more or less returns to its original value. The recovery time of the $\mathrm{SiH}_{3}{ }^{-}$density $\left(\tau_{i}\right)$ is very long (about $600 \mu \mathrm{s}$ at $400 \mathrm{mTorr}$ ), because it depends on the net production rate of $\mathrm{SiH}_{3}{ }^{-}$ions, i.e., the difference between $R_{\text {att }}$ and the ion-ion recombination rate $\left(R_{\text {rcm }}\right)$, and it was shown in Fig. 7 that $R_{\text {att }}-R_{\text {rcm }}$ is very small.

Combining Figs. 9 and 10, we can explain the discrepancy between the measurements and the theoretical results regarding the long time evolution of the negative ion density in Refs. [5,23]. The authors found that the early-time evolution of the local negative ion density is in good agreement with the ballistic approximation equation

$$
n_{-}=n_{-0} e^{-R_{0}^{2} /\left(\nu_{\mathrm{th}} \Delta t\right)^{2}}
$$

where $v_{\text {th }}$ is the thermal velocity of negative ions, $n_{-} 0$ is the steady state value of the local negative ion density $\bar{n}$, and $R_{0}$ is the radius of the laser beam. However, a discrepancy exists at a longer time when the recovery in the measurements is slower than that obtained from the theory. The larger the laser beam diameter, the larger the disagreement. From the recovery behavior of the negative ion density in Figs. 9 and 10, we believe that ignoring the effect of the decrease of the surrounding $\mathrm{SiH}_{3}{ }^{-}$density and ignoring the effect of the inelastic collisions on the recovery of the local negative ion density in Eq. (2) leads to this discrepancy. At the beginning the recovery of the local negative ion density proceeds according to Eq. (2) because the change of the surrounding negative ion density is not important. With the dip being filled up, the surrounding negative ion density de-

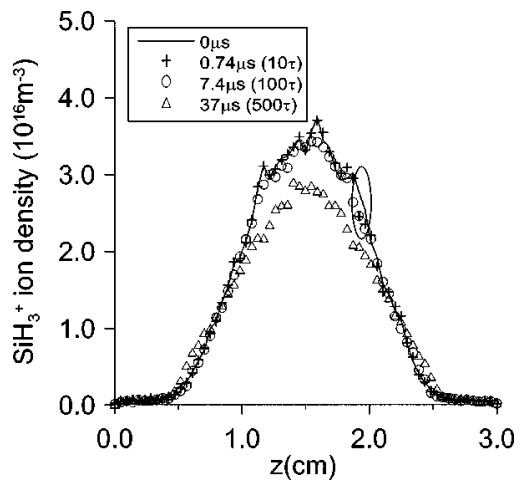

FIG. 11. The calculated evolution of the $\mathrm{SiH}_{3}^{+}$ion density profile after the photodetachment. The conditions are the same as in Fig. 2. The ellipse marks the indirect influence of photodetachment on the positive ion density at the laser impact position.

creases, the gradient of the negative ion density becomes weaker, and, consequently, so does the diffusion. From this point Eq. (2) is not valid anymore. During this period, the recovery of the local negative ion density together with the total negative ion density depends on the net increment rate of $R_{\text {att }}-R_{\text {rcm }}$, which is very small. At larger laser diameters, the influence of the surrounding negative ion density and of the inelastic collisions is larger and the discrepancy appears earlier in time, leading to a larger disagreement between the measured result and the theory.

\section{Relaxation of positive ions $\left(\mathrm{SiH}_{3}{ }^{+}\right)$}

Besides the strong variation of the electron and the negative ion densities, the positive ion density profile $\left(\mathrm{SiH}_{3}{ }^{+}\right)$, as shown in Fig. 11, decreases toward a shape similar to that of the negative ions, due to the requirement of the charge neutrality and due to the reduced production of positive ions by ionization (see Fig. 7). The change appears at the laser impact point (marked in Fig. 11) as well as in the whole discharge. This change reduces the deviation from the neutrality caused by the different diffusion speeds of electrons and negative ions. The change of the local positive ion density was observed indirectly in the experiment (see Fig. 5 of Ref. [6]) by investigating the evolution of the sum of the negative ion density $\left(n_{i-}\right)$ and the extra electron density $\left(\Delta n_{e}\right)$, based on the assumption of charge neutrality (i.e., $n_{i+}$ $\left.=n_{i-}+\Delta n_{e}+n_{e_{-} 0}\right)$. They found that this sum first has a plateau of $600 \mathrm{~ns}$, then decreases in time. It starts to recover when the extra electron density is at the overshoot point.

The full recovery process of the positive ion $\mathrm{SiH}_{3}{ }^{+}$density $\left(n_{i+} / n_{i_{+}} 0\right.$, the ratio of the space and rf period averaged positive ion density and its original value) shown in Fig. 10 behaves similarly to that of the negative ion $\mathrm{SiH}_{3}{ }^{-}$ density. The recovery of $\mathrm{SiH}_{3}{ }^{+}$ions is governed by the net increment of the production by ionization, and the loss by the flow to the wall and the ion-ion recombination. After the electric field and the electrons have nearly completely recovered, this net increment becomes very small, yielding a long recovery time of $\mathrm{SiH}_{3}{ }^{+}$. Note that the curve of $\mathrm{SiH}_{3}{ }^{+}$has a stronger fluctuation than that of $\mathrm{SiH}_{3}{ }^{-}$. This can be ex- 
TABLE I. Calculated recovery time of electrons and ions $\left(\tau_{e}\right.$ and $\tau_{i}$ ) vs photodetachment efficiency, at a pressure of $400 \mathrm{mTorr}$.

\begin{tabular}{crrr}
\hline \hline $\begin{array}{c}\text { Photodetachment } \\
\text { efficiency }(\%)\end{array}$ & 10 & 50 & 100 \\
\hline$\tau_{e}(\mu \mathrm{s})$ & 10 & 17 & 37 \\
$\tau_{i}(\mu \mathrm{s})$ & 170 & 315 & 600 \\
\hline \hline
\end{tabular}

plained by the fact that the ionization reaction only influences $\mathrm{SiH}_{3}{ }^{+}$strongly, and the attachment reaction only influences $\mathrm{SiH}_{3}{ }^{-}$. $R_{\text {ion }}$ has more fluctuation than $R_{\text {att }}$ (see Fig. 7).

From Figs. 3 and 10, one can note a special point in time at about $37 \mu \mathrm{s}$, when the electron density has almost recovered, and the averaged positive ion density and negative ion density reach their minimum. In addition, at this moment, the ballistic approximation for describing the recovery of local negative ion density is no longer satisfied, and the recovery of the local as well as the total negative ion density depends on the net increment rate. This special transition point is also clearly observed in the experimental results (see Fig. 5 of Ref. [6]).

\section{Effect of the photodetachment efficiency and the pressure on the relaxation}

The efficiency of the photodetachment influences the recovery time of the electrons $\left(\tau_{e}\right)$ and ions $\left(\tau_{i}\right)$ strongly. Table I gives the calculated recovery time $\tau_{e}$ and $\tau_{i}$ at a photodetachment efficiency of $10 \%, 50 \%$, and $100 \%$ at 400 mTorr. It is clear that the higher the photodetachment efficiency, the longer the recovery times $\left(\tau_{e}\right.$ and $\left.\tau_{i}\right)$.

Table II gives the pressure effect on $\tau_{e}$ and $\tau_{i}$ with $100 \%$ photodetachment efficiency. It can be seen that with increasing pressure, $\tau_{e}$ rises significantly but $\tau_{i}$ increases only slightly. The recovery time depends on the number of photodetached particles and the recovery speed. Since the charged particle densities do not vary strongly with pressure, the number of photodetached particles increase only slightly compared to the increase of the pressure. The net rate related to the recovery speed of the ions changes also slight, yielding only a minor increase in $\tau_{i}$. The recovery speed of electrons depends on the diffusion speed, as mentioned above. With an increase of the pressure, a correspondingly larger amount of elastic collisions resists the diffusion and hence the recovery time becomes significantly longer.

\section{CONCLUSIONS}

The global relaxation phenomena after instantaneous laser induced photodetachment in an electronegative $\mathrm{SiH}_{4}$ rf dis-

TABLE II. Calculated recovery time of electrons and ions $\left(\tau_{e}\right.$ and $\tau_{i}$ ) vs pressure assuming $100 \%$ photodetachment efficiency

\begin{tabular}{crrr}
\hline \hline Pressure (mTorr) & 30 & 100 & 400 \\
\hline$\tau_{e}(\mu \mathrm{s})$ & 5 & 10 & 37 \\
$\tau_{i}(\mu \mathrm{s})$ & 440 & 490 & 600 \\
\hline \hline
\end{tabular}

charge have been studied by a kinetic 1D PIC-MC model. The effect of the photodetachment on the whole discharge from the perturbation back to equilibrium has been investigated. The evolution of the charged particle densities, the electric field, the EEDF, the reaction rates of the electron impact collisions, and the power dissipation in the whole discharge have been studied in detail. Our results indicate that the relaxation after the local photodetachment has a global character rather than a local character.

Due to the large number of electrons created by the photodetachment, the character of the plasma changes strongly. The change is due to the increase of the electron density which leads to a weaker bulk field, and hence to a drop in the high energy tail of the EEDF and a reduction of the reaction rates of electron impact attachment and ionization. Consequently, the global positive and negative ion densities decrease. All the changes of the variables start from the laser impact point and gradually extend to the whole discharge.

The recovery time for the electrons depends on the electron diffusion speed, while for the ions it depends on the net increment of the production and the loss rate. All variables related directly to the behavior of the electrons, such as the electron density, the electric field, the EEDF, and the reaction rates of the electron impact collisions, recover synchronously to their original values, in hundreds of rf cycles at 400 mTorr. The global recovery of the ion densities starts only after the more or less complete recovery of the electrons. The different recovery mechanisms lead to a recovery time of the ion densities, which is about 1-2 orders of magnitude longer than that of the electrons. The recovery time of the electrons is closely related to the pressure and efficiency of the photodetachment, while the recovery time of the ions is related more to the efficiency of the photodetachment than to the pressure.

The modeled behavior of all the charged particles agrees quite well with experimental results from the literature. The model has been validated for a description of electronegative discharges. In addition, some relaxation processes, which have been implied or guessed in the experiment and previous simulations, have been clarified in our work. The EEDF recovers synchronously with the electron density profile instead of an instantaneous behavior, which was assumed in Refs. [10-13]. The bulk electric field becomes weaker but not stronger after the photodetachment, as assumed in Ref. [6]. The electron density profile, before its recovery to its original form, strongly changes in time as well as in space but not only in time, as assumed in Ref. [1]. The discrepancy in Refs. [5,23] between the measurement and the theory based on the ballistic approximation equation regarding the long-time evolution of the negative ion density is explained by the fact that this approximate equation did not include the influence of the decrease of the surrounding negative ion density and of the inelastic collisions on the recovery of the negative ion density at the laser impact point.

Only a 1D simulation of photodetachment was carried out in this paper, i.e., the laser beam is assumed to be infinite in the directions parallel to the electrodes. However, in actual experiments the laser beam has a finite width; therefore, diffusion in other directions can also influence the results so as 
to yield a shorter recovery time. A $2 \mathrm{D}$ simulation is obviously hampered by the computational effort involved. The present paper is a kind of representative case study of the main relaxation mechanism after photodetachment. A study of relaxation phenomena after photodetachment in other gases mainly requires a specification of a different set of cross sections for the electron-neutral and ion-neutral collisions.

\section{ACKNOWLEDGMENTS}

M.Y. was financed by a New Research Initiative of the University of Antwerp and by the Bilateral scientific and technological cooperation (joint project BIL 99/46) of the Ministry of the Flemish Community, the Ministry of Science and Technology of China, and the National Science Foundation of China. A.B. is indebted to the Flemish Fund for Scientific Research (FWO) for financial support. The PIC-MC code was developed with financial support from The Netherlands Organization for Scientific Research (NWO). The authors also acknowledge financial support from the Belgian Federal Services for Scientific, Technical and Cultural Affairs (DWTC/SSTC) of the Prime Minister's Office through IUAP-IV (Conv. P4/10).
[1] M. Haverlag, A. Kono, D. Passchier, H. M. W. Kroesen, and W. J. Goedheer, J. Appl. Phys. 70, 3472 (1991).

[2] E. Stoffels, W. W. Stoffels, D. Vender, M. Kando, G. M. W. Kroesen, and F. J. de Hoog, Phys. Rev. E 51, 2425 (1995).

[3] R. A. Gottscho and C. E. Gaebe, IEEE Trans. Plasma Sci. PS-14, 92 (1986).

[4] D. Hayashi and K. Kadota, J. Appl. Phys. 83, 687 (1998).

[5] M. Bacal, P. I. Berlemont, A. M. Bruneteau, R. Leroy, and R. A. Stern, J. Appl. Phys. 70, 1212 (1991).

[6] P. Devynck, J. Auvray, M. Bacal, P. Berlemont, J. Bruneteau, R. Leroy, and R. A. Stern, Rev. Sci. Instrum. 60, 2873 (1989).

[7] M. Bacal, Plasma Sources Sci. Technol. 2, 190 (1993).

[8] M. B. Hopkins, M. Bacal, and W. G. Graham, J. Appl. Phys. 70, 2009 (1991).

[9] A. A. Ivanov, A. B. Sionov, F. El Balghiti-Sube, and M. Bacal, Phys. Rev. E 55, 956 (1997).

[10] J. D. P. Passchier and W. J. Goedheer, J. Appl. Phys. 73, 1073 (1993).

[11] M. Shibata, N. Nakano, and T. Makabe, J. Phys. D 30, 1219 (1997).

[12] L. Friedland, C. I. Ciubotariu, and M. Bacal, Phys. Rev. E 49, 4353 (1993).
[13] A. A. Ivanov, L. I. Elizarov, and M. Bacal, Phys. Rev. E 52, 6679 (1995).

[14] A. A. Ivanov, A. B. Sionov, C. I. Ciubotariu, and M. Bacal, Plasma Phys. Rep. 24, 965 (1998).

[15] M. Shibata, N. Nakano, and T. Makabe, J. Appl. Phys. 77, 6181 (1995).

[16] M. Yan and W. J. Goedheer, IEEE Trans. Plasma Sci. 27, 1399 (1999).

[17] C. K. Birdsall, IEEE Trans. Plasma Sci. 19, 65 (1991).

[18] V. Vahedi and M. Surendra, Comput. Phys. Commun. 87, 179 (1995).

[19] E. Kawamura, C. K. Birdsall, and V. Vahedi, Plasma Sources Sci. Technol. 9, 413 (2000).

[20] E. Hamers, Ph.D. thesis, Utrecht University, The Netherlands, 1998.

[21] G. J. Nienhuis and W. J. Goedheer, J. Appl. Phys. 82, 2060 (1997).

[22] J. Perrin, O. Leroy, and M. C. Bordage, Contrib. Plasma Phys. 36, 3 (1996).

[23] R. A. Stern, P. Devynck, M. Bacal, P. Berlemont, and F. Hillion, Phys. Rev. A 41, 3307 (1990). 